\title{
Effect of nicotine exposure during gestation on neonatal rat crystalline lenses
}

C Evereklioglu', B Alaşehirli², I Sari², B Cengiz² and $\mathrm{C}$ Bagci

\section{Abstract}

Purpose To assess histologically the influence of maternal nicotine exposure during gestation in vivo on crystalline lenses in neonatal rats using different dosages of the test compound simulating the range of low, moderate, and heavy smokers in humans. Methods Experimentally naive, adult female Wistar-albino rats (200-250 g) were mated with adult male rats over 2 days for copulation in the proportion of two females for every male animal. After confirming pregnancy with vaginal smear method, 40 gravid rats (dams) were then randomly assigned into four equal groups (three experimental and one control; $n=10$ in each). Groups 1, 2, and 3 experimental dams were treated with intraperitoneal (i.p.) $(-)$-nicotine tartrate at doses of $0.5,1$, and $2 \mathrm{mg} / \mathrm{kg}$ body weight/day, respectively, during pregnancy from gestational days 9 through 21 . Group 4 control dams were given i.p. saline solution daily for the same period. After normal delivery, the eyes were removed at postnatal day 1 or day 30 for macroscopic and histopathologic investigation of the lenses. Results Control and group 1 litters had normal anterior lens capsules with a single layer of anterior cuboidal epithelial cells, regularly orientated cortical and nuclear lens fibres, and a clear posterior lens capsule with no lining epithelial cells behind the equator. On the other hand, some lenses in groups 2 and 3 litters had mature or immature cataract macroscopically at postnatal 30th day. Histopathologic findings suggesting cataractogenesis included cortical lens fibre cell swelling and liquefaction, prominent epithelial cells lining the posterior lens capsule behind the equator, and the retention of lens nuclei into the deeper and central area. Moreover, some lenses were immature developmentally, indicating arrested lenticular embryogenesis at vesicle stage.

Conclusions Nicotine exposure during pregnancy has teratogenic and cataractogenic effects on developing crystalline lenses in neonatal rats both macroscopically and histopathologically. If appropriate dose of nicotine can be identified, nicotine-induced cataract formation may possibly be used as a new experimental cataract model in animal studies. Therefore, future studies are needed in this respect.

Eye (2004) 18, 67-73. doi:10.1038/sj.eye.6700511

Keywords: crystalline lens; neonatal rat; nicotine; pregnancy; teratology

\section{Introduction}

Nicotine, a major active toxic component of tobacco, is a well-known liquid alkaloid obtained from the dried leaves of the tobacco plant, Nicotiana tobacum. Despite decades of adverse publicity, many women all around the world and about one-fourth of all women in the USA and England continue to smoke during pregnancy. ${ }^{1}$ At 10 weeks of gestation, nicotine concentration is five-time higher in embryos than their mothers, and is capable of evoking foetal hypoxia-ischaemia episodes. ${ }^{2}$ Although a biologic causal mechanism has not been clearly delineated, considerable evidences indicate that the chemicals in tobacco pose a unique risk for developmental impairment among children. ${ }^{3,4}$

Human $^{4-7}$ and animal studies ${ }^{8-14}$ have demonstrated that maternal smoking has several detrimental and teratogenic impact on pregnancy and its outcomes such as placental insufficiency, foetal growth retardation, low
${ }^{1}$ Department of Ophthalmology Erciyes University Medical Faculty Kayseri, Turkey

${ }^{2}$ Gaziantep University Medical Faculty Gaziantep, Turkey

Correspondence: C Evereklioglu Sivas Cad.

Cebeci Apt. A-Blok

175/15 Kayseri, TR-38020 Turkey

Tel.: + 903522331565

Fax: + 903522358365

E-mail: evereklioglu@

hotmail.com

Web: http://

www.evereklioglu.8m.com

Received: 2 October 2002 Accepted in revised form: 31 December 2002 
birth weight, foetal brain damage, microcephaly, anancephaly, cleft palate, impaired skin and alveolar maturation, ultrastructural testicular changes, intracranial haemorrhage, increased perinatal morbidity, spontaneous abortion with perinatal deaths, and sudden infant death syndrome. In addition, the effect on lactation and, therefore, on surviving children as long-term learning disabilities, disruptive behaviour, and attention deficit with hyperactivity disorder are all much more common in the offspring of women who smoke. ${ }^{7,15}$

The only known eye disease that smoking has been implicated in is a bilateral optic neuropathy, namely, tobacco-toxic optic neuropathy. ${ }^{16}$ Our more recent clinical study have demonstrated that chronic smoking in healthy subjects has detrimental effects on retinal and optic nerve functions. ${ }^{17}$ Epidemiological studies have shown that smoking is, however, a major considerable risk factor for strabismus, ${ }^{18}$ thyroid ophthalmopathy, ${ }^{19}$ and cataract. ${ }^{20}$ Therefore, it is important to know whether nicotine is actually teratogenic on the eye during embryofoetal organogenesis. Considering the toxicological risks of nicotine claimed by many different groups of teratologists, this experimental investigation aimed for the first time to explore whether nicotine had any effect on embryofoetal lenticular development during gestation in neonatal rats macroscopically or histopathologically.

\section{Materials and methods}

Appropriate permits for the study were obtained from the Animal Experimental Ethics Committee at the university. The experiments conformed to the Council Directive of the European Communities. Rats were obtained from the Medical Science Research and Application Centre of the university.

\section{Animal and tissue preparations}

Experimentally naive, 40 adult virgin Wistar-Albino rats (200-250 g) were acclimated to caged laboratory conditions for 2 weeks. They were allowed to feed with standard pellet during the study. Rats were housed in stainless-steel cages in a temperature- and humiditycontrolled room with a 12-h light/dark cycle (7:0019:00 $\mathrm{h}$ lights on). Female rats were mated with adult male rats over 2 days for copulation in the proportion of two female animals for every male animal. After confirming pregnancy with vaginal smear method, ${ }^{13}$ gravid rats (dams) were then randomly assigned into four equal groups (10 in each group), three experimental and one control.

On day 9 of gestation, a day critical for the development of teratologic effects in this species, ${ }^{13,21,22}$ experimental dams were treated with intraperitoneal (i.p.) nicotine ((-)-nicotine tartrate, ICN Biomedicals Inc., $\mathrm{OH}, \mathrm{USA}$ ) at doses of $0.5 \mathrm{mg} / \mathrm{kg}$ body weight/day (group $1, n=10$ ), $1 \mathrm{mg} / \mathrm{kg}$ body weight/day (group 2 , $n=10$ ), and $2 \mathrm{mg} / \mathrm{kg}$ body weight/day (group $3, n=10$ ) during pregnancy from 9 through 21 days of gestation. The given doses and the route of nicotine throughout gestation are the regimens that produce plasma nicotine levels spanning the range of low, moderate, and heavy smokers in humans, respectively. ${ }^{23,24}$ Control dams (group 4, $n=10$ ) were injected i.p. saline solution daily during pregnancy for the same period. Clinical status of the dams was monitored daily during nicotine treatment and all groups were examined daily using ultrasonography (US). A real-time US (Toshiba SSA-270A) and 7.5 MHz linear transducer were used to follow and count the number of foetuses.

All dams were allowed normal delivery during the 20th or 21st day of gestation and maternal and offspring body weights as well as the number and the crownrump lengths of the litters were all recorded in each group at birth. Half of the litters in each group was then killed by decapitation, after which the eyes were enucleated and examined for morphological characteristics.

\section{Histology}

The globes were separately numbered, fixed in a solution of $10 \%$ formaldehyde, and prepared for the histological examination. The tissues were then embedded in paraffin wax, sections of 4-6 $\mu \mathrm{m}$ thickness were obtained, mounted on slides and stained with haematoxylin-eosin for routine light microscopy. The slides were histologically investigated by a physician, masked as to the treatment group. Multiple sections from paraffin blocks were performed to obtain the desired lens section with maximal anteroposterior diameter, so that the section would pass from the middle of the lens, not periphery. Other half of litters in each group was left in their cages, all biological mothers raised their own litters, and then were killed by decapitation at postnatal 30th day for macroscopic, biomicroscopic, and histopathologic examination of the lenses as in the first evaluation.

\section{Statistics}

Data were analysed by ANOVA and multiple comparison procedures based on Tukey-HSD method as indicated. Statistical analysis was performed with Statistical Package for the Social Sciences for Windows (SPSS Inc., version 7.5, Chicago, IL, USA). 


\section{Results}

\section{Clinical results}

Nicotine-exposed experimental dams in group 3 showed some signs of distress and irritability such as hyperactivity and anger immediately after nicotine administration. There were five resorbed and two stillborn foetuses in group 3 treated with $2 \mathrm{mg} / \mathrm{kg}$ body weight/day of nicotine. Therefore, the average number of litters was lower in experimental group 3 (mean number, 8.3) than control group (mean number, 9.0), although the difference was not significant $(P>0.05)$. Maternal nicotine exposure caused reduced maternal weight gain during gestation in group $1(11 \% ; P>0.05)$, group 2 (23\%; $P<0.05)$, and group 3 (39\%; $P<0.01)$ when compared with control animals (data not given). In addition, offspring birth weight was reduced by $5 \%$ $(P>0.05), 13 \%(P<0.05)$ and $16 \%(P<0.05)$, respectively, when compared with control litters. Moreover, mean crown-rump length of the offspring was also reduced accordingly in groups 2 and 3, but the difference was only significant for group 3 compared with controls (mean \pm SD; $3.5 \pm 0.3$ vs $4.0 \pm 0.2 \mathrm{~cm} ; P<0.05$ ). Since many previous studies evaluated in detail and it was out of the scope of this study, we did not concentrate on the other possible developmental changes in dams and in offspring, but eyes. Macroscopically visible gross ocular anomaly could not be observed externally in both the experimental groups and control group of neonatal litters.

\section{Histopathological results}

Both macroscopic and histopathologic examinations of the lenses by light microscope in the control group demonstrated normal findings with a regularly shaped single layer of anterior cuboidal epithelial cells, regularly arranged cortical and nuclear lens fibres, peripherally located lens fibre nuclei, and a clear posterior lens capsule without any lining epithelial cells behind the equator (Figures 1a and b). Similar findings were also observed in the lenses of group 1 litters. On the other hand, some litters in group 3 treated with higher dose of nicotine developed externally visible mature cataract at postnatal 30th day (Figure 2). Many of the remaining lenses in both groups 2 and 3, however, demonstrated striking histopathological changes suggesting cataractogenesis. The most common finding was irregularly oriented lens fibres with liquefaction, and the presence of a number of swollen fibre cells both in the anterior and posterior cortexes (Figure 3). In addition, another conspicuous finding was the prominent epithelial cells lining the posterior lens capsule behind the equator with retention of nuclei into the central
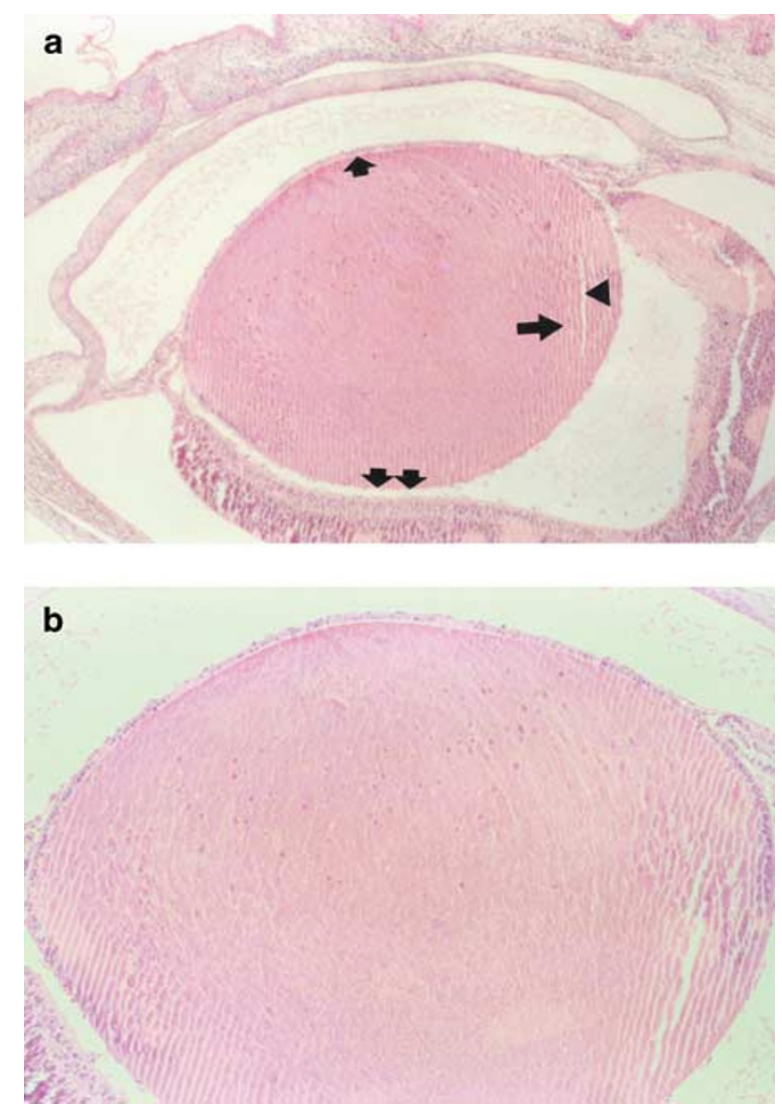

Figure 1 (a) In the control group of neonatal rats, a normal crystalline lens with a regularly shaped single layer of anterior cuboidal epithelial cells (short arrow), clear and regularly arranged cortical and nuclear lens fibres (long arrow) with clear posterior lens capsule without lining epithelial cells behind the equator (double arrow). Lenticular fragmentation is a histologic artefact (arrowhead) (H\&E, original magnification $\times 40$ ). (b) Higher magnification of a for the details ( $\mathrm{H} \& \mathrm{E}$, original magnification $\times 200$ ).

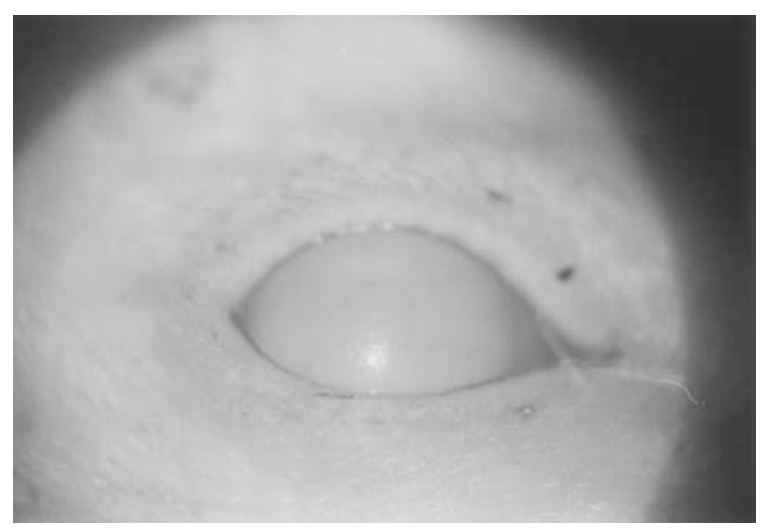

Figure 2 In the experimental group 3 of neonatal rats, macroscopic finding of mature cataract at postnatal 30th day (it was bilateral). 


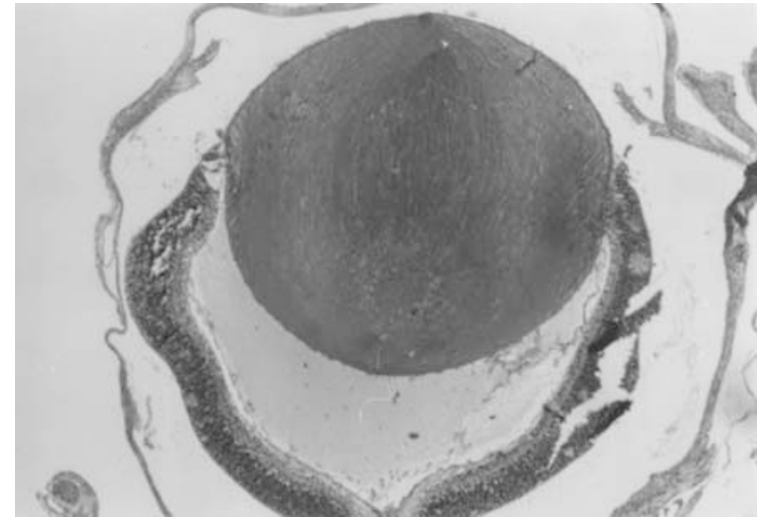

Figure 3 In the experimental group of neonatal rats, irregularly oriented lens fibres, and quite a number of swollen fibre cells with some liquefaction, especially in the posterior lens cortex $(\mathrm{H} \& \mathrm{E}$, original magnification $\times 100)$.

region (Figures $4 a$ and $b$ ). Furthermore, some of the lenses were morphologically immature and defective, suggesting an arrest during ocular embryogenesis at the lens vesicle stage (Figure 5). Taken together, $57 \%$ of the lenses in groups 2 and 3 demonstrated at least one of the aforementioned histopathologic or macroscopic changes.

\section{Discussion}

Nicotine has a low molecular weight and is absorbed after inhalation by buccal and nasal mucosa, alveoles, and by skin contact. ${ }^{25}$ Nicotine exposure during pregnancy by active or passive cigarette smoke is likely to be the single most widespread chemical insult in the world and is a common public health problem, continuing unabated despite decades of educational and medical intervention. It is likely to become of even greater significance with the increasing use of tobacco in the Third World countries that have typically high pregnancy rates. Furthermore, the increase in tobacco use by younger women than ever before will continue to play an important role in the effects of smoking to embryofoetal and neonatal development.

Smokers tend to maintain plasma nicotine levels at a constant value and tobacco/nicotine is capable of evoking acute episodes of foetal hypoxia-ischaemia. It is an anorexic drug and thus influences the maternal nutritional state. In addition, the effect is long-lived and persists into the second postnatal week. ${ }^{26}$ These actions of nicotine on the foetus does not necessarily depend on the effect on placental blood flow and maternal or foetal blood pressure, but may also be a direct one as a result of nicotine delivery itself, representing a true cause-effect
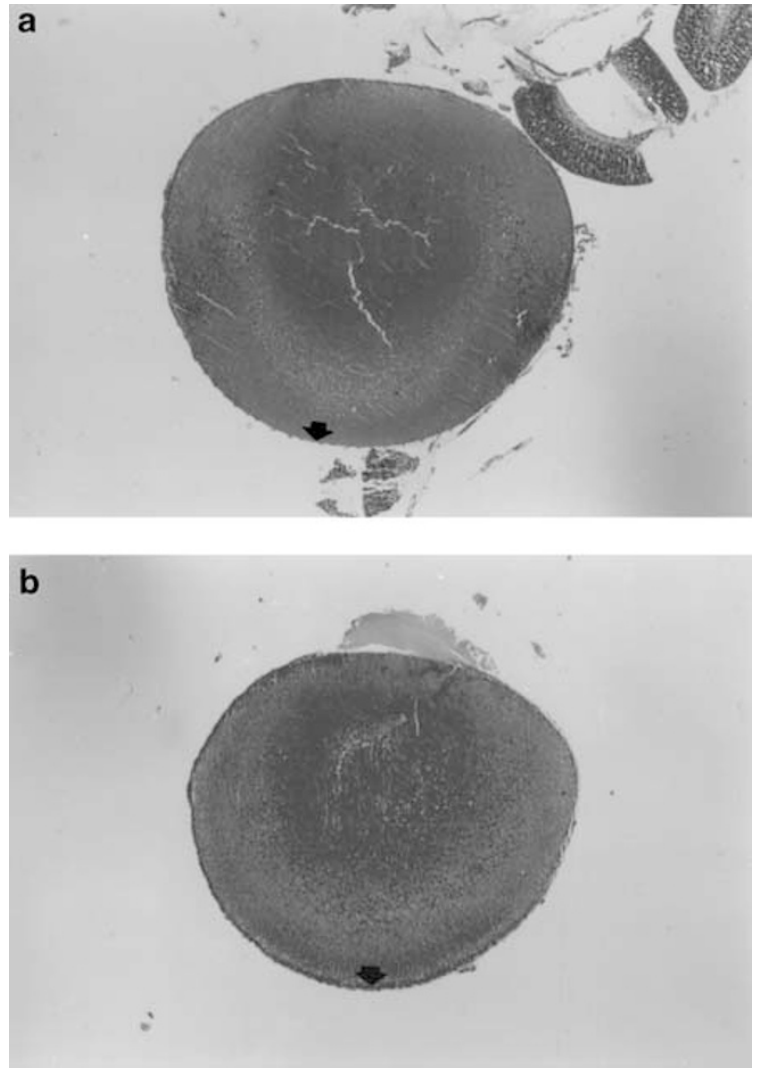

Figure 4 In the experimental group 3 of neonatal rats, two different lenses of two siblings at postnatal day 1. (a) Lenticular fragmentation can be a histologic artefact, however, the posterior lens capsule is normally clear with no lining epithelial cells (short arrow) (H\&E, original magnification $\times 100)$. (b) This is the lens of the sibling shown in a with the prominent epithelial cells lining the posterior lens capsule behind the equator (short arrow) and retention of nuclei into the central region simulating the cataracts of rubella embryopathy (H\&E, original magnification $\times 100$ ).

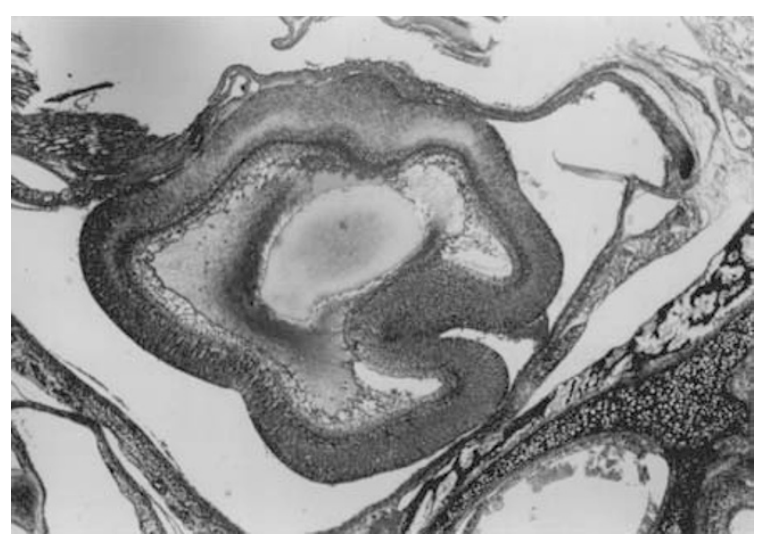

Figure 5 In the experimental group 3 litters, an immature and defective lens, indicating a very early disruption of lenticular embryogenesis at the lens vesicle stage (H\&E, original magnification $\times 40$ ). 
relation. ${ }^{24,26}$ Cigarette smoking exposes the foetus to both nicotine and hypoxia/ischaemia not only during pregnancy but at postnatal period by second-hand smoke, which involves substances that cause hypoxia like $\mathrm{CO}$ and $\mathrm{HCN}{ }^{27}$

Histologically, van der Veen and Fox ${ }^{28}$ have demonstrated that there is apparently an impairment in placental function caused by placental ischaemia in cigarette smokers. Human foetuses are also exposed to significant levels of nicotine with a five-fold increase in the incidence of sudden infant death syndrome among infants born to smoking mothers. ${ }^{24,29}$ Nicotine is a neuroteratogen, acting to cause cell damage and reduced cell number during embryofoetal development, and at postnatal first 2 weeks. ${ }^{26}$ Furthermore, congenital cataracts are responsible for up to $40 \%$ of blindness in children. ${ }^{20}$ Therefore, since smoking mothers expose their infants to nicotine with numerous detrimental and teratogenic effects stated above, this study investigated whether maternal nicotine exposure during gestation affects neonatal lenticular development. We gave nicotine during organogenesis period with the regimens that produce plasma nicotine levels simulating the range in human smokers. ${ }^{12}$ The dose and route of nicotine treatment used in the present study were similar with those in previous experimental studies from 0.25 through $4 \mathrm{mg} / \mathrm{kg}$ body weight/day. ${ }^{12-14,23,24}$

Histologically, the anterior subcapsular epithelial monolayer normally terminates at the lens equator, and thin posterior lens capsule is normally clear with no lining epithelial cells in haematoxylin-eosin-stained preparations. Posterior subcapsular cataract results from the migration of the lens epithelial cells over the posterior lens capsule behind the equator. ${ }^{30}$ This change was clearly observed in some of our lenses in the offspring of experimental groups. It could be suggested that lens epithelial cells might be damaged by toxic response, causing cataractous development by distribution anomalies of the epithelial cells. In addition, as the peripheral lens fibres become displaced toward the centre of the lens, their nuclei normally disintegrate so that central area lacks nuclei. Teratogenic effect of congenital diseases, like rubella, is characterised by the persistence of the lens epithelial nuclei within the lens fibres in the deeper and central area. ${ }^{31}$ This finding was also present in our lenses in experimental group treated with higher dose of nicotine.

Cortical cataract results from the degeneration, destruction, and liquefaction of the cortical lens fibre cells. ${ }^{31}$ In its incipient stage, the formation of vacuoles, globules, or clefts in the lens cortex is observed. Then, interrupted and folded lens fibres and cortical clefts are filled with morgagnian globules, degenerated lens cortex swells by osmotic effect, and total cortical liquefaction eventually ensues. Such histopathologic changes were demonstrated by Rogers and Grabowski ${ }^{32}$ on mirexinduced cataractogenesis in perinatal rats. In the present study, these findings were considerable with marked liquefaction and destruction of the cortical lens fibres in the anterior and posterior poles. In addition, there were quite a number of irregularly oriented swollen cortical lens fibres. Therefore, these findings indicated a lens with a cortical and complicated cataract.

At postnatal day 30, some lenses of our litters developed immature or mature cataract macroscopically. Furthermore, some lenses were developmentally immature, suggesting arrested maturation at the lens vesicle stage. ${ }^{30,31}$ Taken together, these changes suggested that nicotine might initiate a change in the programme for lenticular cell development and might ultimately lead to cell loss as a result of apoptosis by the inhibition of DNA synthesis in foetus and neonates. ${ }^{26,33}$ Therefore, this might have caused developmental abnormality with an early arrest in the lens cell differentiation.

A potential role of cigarette smoking in cataractogenesis has been shown by a series of epidemiological studies in England ${ }^{34,35}$ and the USA. ${ }^{36,37}$ The relative risk for cataract ranged from 1.0 for past light smokers to 2.6 for past heavy smokers, to 2.9 for present heavy smokers. ${ }^{35}$ The other study found the relative risk of cataract in heavy smokers as 2.1 compared with exsmokers and nonsmokers. ${ }^{37}$ Despite a number of epidemiological papers that have linked cataract with smoking, there is still no clear understanding of the extent to which smoking in general, and various components of cigarette smoke in particular, exerts a detrimental effect on human cataract. Likewise, there is also no other study evaluating directly the effect of nicotine on embryofoetal lenticular development histopathologically. Therefore, this study is the first investigation exploring the possible teratogenic and cataractogenic effects of nicotine in vivo on neonatal rat lenses experimentally.

Some speculations about the mechanisms of nicotine effect may be hypothesised. Nicotine-induced vasoconstriction of the placental vessels and decreased capillary blood flow during pregnancy may result in the hypoxic-ischaemic necrosis of the nourished tissues, including the lenses. Likewise, smoking may induce oxidative stress by the increased production of oxygenderived free radicals including superoxide anion, which may overweight the partial inhibition of antioxidants against the damage. ${ }^{38}$ Some of these changes, however, cannot be explained only on this basis and it can be suggested that this may possibly be due directly to nicotine (a toxic alkaloid) and its metabolite cotinine toxicity or to the accumulation of polycyclic aromatic hydrocarbons. ${ }^{39}$ In addition, cadmium (a heavy metal) 
may also participate in this process. In this respect, Bush et $a l^{40}$ have shown that the most significant alterations of maternal cigarette smoking are increased cadmium levels within the placenta. Moreover, Cekic ${ }^{41}$ has clearly demonstrated in his recent study on human cataractous lenses that cigarette smoking may be cataractogenic by lenticular copper, lead, and cadmium accumulation, even in moderate smokers. The other suggested mechanism for nicotine action may be the direct effect of highly lipid soluble teratogens on the membranes by lipid peroxidation with generation of toxic products within the eye or indirect by oxidative damage. ${ }^{42}$ Some other trace metals in the blood may also be responsible for cataractogenesis. ${ }^{43}$ Taken together, smoke-induced damage possibly occurs through systemic absorption and transport of these toxic components to several tissues, specially into the lens, wherein the turnover is slow. This possibly leads to the chronic accumulation of toxic components of tobacco and, therefore, oxidative damage to the constituent molecules, resulting in lenticular opacity. Our recent clinical study in smokers have also suggested this hypothesis and demonstrated impairments in retinal and optic nerve functions. ${ }^{17}$

In conclusion, this was the first investigation that examined the effect of nicotine exposure during pregnancy on neonatal rat lenses at levels simulating low, moderate, and heavy smokers in humans. We histopathologically demonstrated that the effects of gestational nicotine exposure on developing embryofoetal crystalline lenses were clear in the offsprings. Although many findings were conspicuous for cataractogenesis in the lenses at postnatal first day, immature and mature cataracts were, in turn, observed by biomicroscopic examinations at postnatal 30th day in the litters treated with higher dose of nicotine. Applying animal data directly to humans requires restrain. However, it can be suggested that a pregnant woman as well as nursing mothers should be seriously warned in consuming nicotine during pregnancy for healthy embryofoetal lenticular development, because the effectiveness of filter tips for reducing nicotine, cadmium and $\mathrm{CO}$ in cigarettes is insufficient. Moreover, these effects can be reversed if they give up smoking in pregnancy or suckling. The second and third trimesters are more sensitive to adverse effects of nicotine than the first trimester. Therefore, future studies will help us to clarify the significance between the trimester and smoking. Likewise, possible lenticular effects of nicotine on lactation and long-term effects on surviving litters are also to be specified. Furthermore, nicotine-induced cataract formation can be used as a new experimental cataract model in animal studies, if appropriate doses are identified.

\section{Acknowledgement}

Authors have no financial or proprietary interest in any instrument or products used in this study.

\section{References}

1 Bardy AH, Seppala T, Lillsunde P, Kataja JM, Koskela P, Pikkarainen $\mathrm{J}$ et al. Objectively measured tobacco exposure during pregnancy: neonatal effects and relation to maternal smoking. Br J Obstet Gynaecol 1993; 100: 721-726.

2 Luck W, Nau H, Hansen R, Steldinger R. Extent of nicotine and cotinine transfer to the human fetus placenta and amniotic fluid of smoking mothers. Dev Pharmacol Ther 1985; 8: 384-395.

3 Walsh RA. Effects of maternal smoking on adverse pregnancy outcomes: examination of the criteria of causation. Hum Biol 1994; 66: 1059-1092.

4 Cnattingius S, Haglund B, Meirik O. Cigarette smoking as risk factor for late fetal and early neonatal death. BMJ 1988; 297: 258-261.

5 Olsen J. Cigarette smoking in pregnancy and fetal growth. Does the type of tobacco play a role? Int J Epidemiol 1992; 21: 279-284.

6 DiFranza JR, Lew RA. Effect of maternal cigarette smoking on pregnancy complications and Sudden Infant Death Syndrome. J Fam Pract 1995; 40: 385-394.

7 Huisman M, Risseeuw B, van Eyck J, Arabin B. Nicotine and caffeine. Influence on prenatal hemodynamics and behavior in early twin pregnancy. J Reprod Med 1997; 42: 731-734.

8 Abel EL. Smoking during pregnancy: a review of effects on growth and development of offspring. Hum Biol 1980; 52: 593-625.

9 Riesenfeld A, Oliva H. Effects of nicotine on the fertility, cytology and life span of male rats. Acta Anat 1988; 131: 171-176.

10 Joschko MA, Dreosti IE, Tulsi RS. The teratogenic effects of nicotine in vitro in rats: a light and electron microscope study. Neurotoxicol Teratol 1991; 13: 307-316.

11 Forrest CR, Pang CY, Lindsay WK. Pathogenesis of ischemic necrosis in random-pattern skin flaps induced by long-term low-dose nicotine treatment in the rat. Plast Reconstr Surg 1991; 87: 518-528.

12 Maritz GS, Dennis H. Maternal nicotine exposure during gestation and lactation interferes with alveolar development in the neonatal lung. Reprod Fertil Dev 1998; 10: 255-261.

13 Inaloz HS, Inaloz SS, Deveci E, Eralp A. Teratogenic effects of nicotine on rat skin. Clin Exp Obstet Gyn 2000; 27: 241-243.

14 Aydos K, Guven MC, Can B, Ergun A. Nicotine toxicity to the ultrastructure of the testis in rats. BJU Int 2001; 88: 622-626.

15 Eskenazi B, Trupin LS. Passive and active maternal smoking during pregnancy, as measured by serum cotinine, and postnatal smoke exposure. II. Effects on neurodevelopment at age 5 years. Am J Epidemiol 1995; 142: 19-29.

16 Rizzo III JF, Lessell S. Tobacco amblyopia. Am J Ophthalmol 1993; 116: 84-87.

17 Hepsen IF, Evereklioglu C. Defective visual field tests in chronic heavy smokers. Acta Ophthalmol Scand 2001; 79: $53-56$. 
18 Hakim RB, Tielsch JM. Maternal cigarette smoking during pregnancy. A risk factor for childhood strabismus. Arch Ophthalmol 1992; 110: 1459-1462.

19 Hamilton SR. Neuro-ophthalmology of eye-movement disorders. Curr Opin Ophthalmol 1999; 10: 405-410.

20 Ojofeitimi EO, Adelekan DA, Adeoye A, Ogungbe TG, Imoru AO, Oduah EC. Dietary and lifestyle patterns in the aetiology of cataracts in Nigerian patients. Nutr Health 1999; 13: 61-68.

21 Lichtensteiger W, Ribary U, Schlumpf M, Odermatt B, Widmer HR. Prenatal adverse effects of nicotine on the developing brain. Prog Brain Res 1988; 73: 137-157.

22 Roy TS, Andrews JE, Seidler FJ, Slotkin TA. Nicotine evokes cell death in embryonic rat brain during neurulation. J Pharmacol Exp Ther 1998; 287: 1136-1144.

23 Tolson CM, Seidler FJ, McCook EC, Slotkin TA. Does concurrent or prior nicotine exposure interact with neonatal hypoxia to produce cardiac cell damage? Teratology 1995; $\mathbf{5 2}$ 298-305.

24 Slotkin TA, Lappi SE, McCook EC, Lorber BA, Seidler FJ. Loss of neonatal hypoxia tolerance after prenatal nicotine exposure: implications for sudden infant death syndrome. Brain Res Bull 1995; 38: 69-75.

25 Benowitz NL, Lake T, Keller KH, Lee BL. Prolonged absorption with development of tolerance to toxic effects after cutaneous exposure to nicotine. Clin Pharmacol Ther 1987; 42: 119-120.

26 Slotkin TA. Fetal nicotine or cocaine exposure: which one is worse? J Pharmacol Exp Ther 1998; 285: 931-945.

27 Hutchison SJ, Glantz SA, Zhu BQ, Sun YP, Chou TM, Chatterjee $\mathrm{K}$ et al. In utero and neonatal exposure to secondhand smoke causes vascular dysfunction in newborn rats. J Am Coll Cardiol 1998; 32: 1463-1467.

28 van der Veen F, Fox H. The effects of cigarette smoking on the human placenta: a light and electron microscopic study. Placenta 1982; 3: 243-256.

29 Clegg DA, O'Hara BF, Heller HC, Kilduff TS. Nicotine administration differentially affects gene expression in the maternal and fetal circadian clock. Brain Res Dev Brain Res 1995; 84: 46-54.

30 Klintworth GK, Garner A. The causes, types, and morphology of cataract. In: Garner A, Klintworth GK (eds). Pathology of Ocular Disease: A Dynamic Approach, 2nd ed. Marcel Dekker: New York, 1994: 2211-2255.
31 Klintworth GK, Eagle Jr RC. The eye and ocular adnexa. In: Stephen SS (ed). Diagnostic Surgical Pathology, 3rd ed. Lippincott Williams \& Wilkins: Philadelphia, PA, 1999 pp 975-1007.

32 Rogers JM, Grabowski CT. Postnatal mirex cataractogenesis in rats: lens cation balance, growth and histology. Exp Eye Res 1984; 39: 563-573.

33 McFarland BJ, Seidler FJ, Slotkin TA. Inhibition of DNA synthesis in neonatal rat brain regions caused by acute nicotine administration. Brain Res Dev Brain Res 1991; 58: 223-229.

34 Harding JJ, van Heyningen R. Beer, cigarettes and military work as risk factors for cataract. Dev Ophthalmol 1989; 17: 13-16.

35 Flaye DE, Sullivan KN, Cullinan TR, Silver JH, Whitelocke RA. Cataracts and cigarette smoking. The City Eye Study. Eye 1989; 3: 379-384.

36 West S, Munoz B, Emmett EA, Taylor HR. Cigarette smoking and risk of nuclear cataracts. Arch Ophthalmol 1989; 107: 1166-1169.

37 Christen WG, Manson JE, Seddon JM, Glynn RJ, Buring JE, Rosner B et al. A prospective study of cigarette smoking and risk of cataract in men. JAMA 1992; 268: 989-993.

38 Shalini VK, Luthra M, Srinivas L, Rao SH, Basti S, Reddy M et al. Oxidative damage to the eye lens caused by cigarette smoke and fuel smoke condensates. Indian J Biochem Biophys 1994; 31: 261-266.

39 Zenzes MT. Smoking and reproduction: gene damage to human gametes and embryos. Hum Reprod Update 2000; 6: 122-131.

40 Bush PG, Mayhew TM, Abramovich DR, Aggett PJ, Burke MD, Page KR. A quantitative study on the effects of maternal smoking on placental morphology and cadmium concentration. Placenta 2000; 21: 247-256.

41 Cekic O. Effect of cigarette smoking on copper, lead, and cadmium accumulation in human lens. $\mathrm{Br} J$ Ophthalmol 1998; 82: 186-188.

42 Zigler Jr JS, Bodaness RS, Gery I, Kinoshita JH. Effects of lipid peroxidation products on the rat lens in organ culture: a possible mechanism of cataract initiation in retinal degenerative disease. Arch Biochem Biophys 1983; 225: 149-156.

43 Doganay S, Turkoz Y, Evereklioglu C, Er H, Bozaran M, Ozerol E. Use of caffeic acid phenethyl ester to prevent sodium-selenite-induced cataract in rat eyes. J Cataract Refract Surg 2002; 28: 1457-1462. 